\title{
EL APORTE DE LA BIOÉTICA A LAS INSTITUCIONES PÚBLICAS EN SALUD: EXPERIENCIAS DEL ÁREA DE BIOÉTICA MINISTERIO DE SALUD DE CÓRDOBA (ARGENTINA)
}

\section{THE CONTRIBUTION OF BIOETHICS TO PUBLIC HEALTH INSTITUTIONS: EXPERIENCES OF THE BIOETHICS AREA OF THE MINISTRY OF HEALTH OF CÓRDOBA (ARGENTINA)}

\section{María Fernanda Marchetti ${ }^{1}$ - Andrés F. Toledo ${ }^{2}$}

DOI: https://doi.org/10.37767/2591-3476(2021)01

En 1998, fue creada el Área de Bioética, dependiendo en la actualidad de la Secretaría de prevención y promoción de salud del Ministerio de Salud de la Provincia de Córdoba. Argentina.

A lo largo de estos años, ha ido evolucionado en distintos objetivos y competencias en el campo disciplinar de la bioética. En la actualidad, los objetivos y funciones del Área de Bioética han sido definidos por la Resolución Ministerial № 1264, con su anexo 1, de fecha 23 de noviembre de 2017.

En todo este tiempo, pasando por distintos momentos sociales y políticos, el Área de Bioética desarrolló distintas líneas de trabajo tomando como base aquellas que se marcan como las funciones principales de los comités de bioética a nivel de meso gestión, que son las funciones educativas, consultivas y normativas (Tealdi et al, 1990). De hecho, a partir de un trabajo sostenido de coordinación y acompañamiento se pudo constituir la "Red de comités hospitalarios de bioética" de la provincia que es uno de los principales frutos de todos estos años de trabajo.

Esta presencia permanente en bioética ha servido y sirve para generar una línea de formación y capacitación tomando como base una reflexión actualizada de los derechos humanos ya consagrados, y de manera particular la declaración de bioética y derechos

1 Coordinadora del Área de Bioética Ministerio de Salud. Provincia de Córdoba. Médica, especialista en medicina paliativa, experta en bioética. Mail: fermarchetti@hotmail.com. ORCID iD: https://orcid.org/0000-0001-5503-0627.

2 Equipo permanente Ârea de Bioética Ministerio de Salud. Provincia de Córdoba. Doctor en Filosofía, Magister en Bioética y Salud Pública. Mail: andrestoledo14@hotmail.com. ORCID iD: https://orcid.org/0000-0002-1175-8568 . 
humanos de UNESCO 2005. Con estos marcos teóricos axiológicos se trata de abordar de una manera interdisciplinar los problemas sanitarios más urgentes de nuestra región como la inequidad social existente. los problemas de accesibilidad en salud, entre otros.

Esta convicción de reflexión y acción es lo que se expresa en- la página web del Área de Bioética donde se rescata el compromiso por todas las realidades especialmente marcadas por la exclusión y que requiere no solamente una reflexión atinada, sino también acciones concretas que ayuden a cambiar las realidades de nuestro contexto social sanitario.

En nuestra página web se lee:

El "Área de Bioética", ha sostenido y sostiene, la bioética como una disciplina y como un movimiento; que, de reciente aparición en el concierto de las ciencias y de las humanidades (1971), se ha alzado y pretende ser, el lugar de encuentro de dos grandes aspiraciones del hombre: como es, el progreso de sus inventivas tecnológicas; y de buscar cada día, "vivir mejor", o contar con mejor calidad de vida.

Lo importante, para el "Área de Bioética", ya sea la bioética como disciplina o como movimiento; es que sea una bioética comprometida, y que cada acción o reflexión que se realice busque aportar para hacer un mundo más justo y un lugar más humano. (https://www.cba. gov.ar/area-de-bioetica/)

\section{A nivel educativo/formativo}

Una de las principales actividades del Área de Bioética en el plano educativo es la organización del "Seminario Taller de Bioética Clínica Social", espacio de aprendizaje colaborativo que desde hace 20 años viene sosteniendo y que se ha convertido en un lugar de formación de excelencia y de encuentro de redes de distintos interesados en la bioética, tanto a nivel público como privado.

Sin ser exhaustivos y a modo de ejemplo, señalamos algunas de nuestras actividades actuales en el plano educativo.

- Curso Virtuales. Desde el 2018 se sostiene y fortalece esta iniciativa en la plataforma moodle (https://areabioetica.milaulas.com/) con un total de 1200 inscriptos donde 500 participantes han egresado porque realizaron su correspondiente examen de aprobación. Los cursos tienen el objetivo central de brindar formación gratuita, de calidad y especializada en esta materia. Se destaca que tenemos muchos alumnos/ as extranjeros.

- Capacitaciones cortas para agentes sanitarios de la provincia. En estos años se realizaron varias capacitaciones en bioética en ámbitos relacionados a la salud como encuentros con enfermeros/as de la Municipalidad de Córdoba, ateneos en distintos hospitales, charlas dentro de jornadas científicas interdisciplinarias, clases a residentes y encuentros en colegios secundarios de la provincia.

- Creación del blog del Área de Bioética http://areadebioetica.blogspot.com.ar/) y canal You Tube (https://www.youtube.com/channel/UCXmL74ZMEKYbanAq216b9mw) donde no solamente se brinda información de distintas actividades de bioética sino también videos y charlas grabadas de temas actuales de bioética con temas como perspectiva de género y bioética, etc.

- Dos actividades centrales dentro de la agenda anual son la organización y realización de las "Jornadas Provinciales de Bioética" cada dos años y la "Semana Provincial de Bioética". En ambos espacios se intenta visibilizar la bioética desde la tarea que reali- 
zan los distintos comités hospitalarios de bioética de la provincia y generar espacios de encuentro de formación y de actividades de consolidación de redes sanitarias.

- Articulación con otras instituciones y grupos que trabajan en bioética como: Universidad Nacional de Córdoba. Instituto Universitario de Ciencia Biomédico Córdoba. Universidad Católica de Córdoba., Consejo de Médicos de Córdoba y entidades de la sociedad civil donde se ofrecen capacitaciones conjuntas.

\section{A nivel consultivo}

En este nivel el Área de Bioética interactúa constantemente con distintas entidades que formulan consultas sobre los más variados temas como: organismos de coordinación de trasplante, mesas de trabajo en salud pública convocadas por el Ministerio de Salud, o consultas de temas muy delicados sobre inicio y final de vida que se dan en nuestras instituciones hospitalarias y que requieren una palabra de nuestra Área. Un tema de constante consulta ha sido la provisión de medicamentos de alto costo desde una mirada del principio de justicia y solidaridad.

También en algunas ocasiones ha sido solicitado la participación del Área para contestar requerimientos judiciales.

Esta función consultiva tan propia de los comités hospitalarios, en esta área central tiene las características de acompañamiento a las recomendaciones que dan los comités institucionales y cuando se trata de temas más generales es donde el Área brinda su servicio y trata de deliberar sobre los mejores cursos de acción en bioética.

\section{A nivel normativo}

En este nivel el Área de Bioética más que generar normas propone indicaciones, guías sobre distintos temas de salud y bioética. Se señalan algunos trabajos recientes: confección de una lista de derechos y responsabilidades de los pacientes para los hospitales públicos, también ha confeccionado un modelo de consentimiento informado general para internación e ingreso a las instituciones hospitalarias. En un nivel institucional también confeccionó los reglamentos internos de funcionamiento de los distintos comités hospitalarios de bioética.

\section{Participación del Área de Bioética durante la pandemia por Covid-19}

Lo vivido en esta pandemia determinó una serie de problemas novedosos, pero sobre todo dejó en evidencia muchos viejos problemas de nuestra región como la desigualdad, la pobreza, las deficiencias en los sistemas de salud, etc. Esto motivó que desde la bioética se sintiera la necesidad de aportar una voz que busca optimizar resultados y beneficios para la mayoría de la sociedad, rescatar el valor de la solidaridad y la responsabilidad individual y social.

Desde el Área de Bioética se pudo participar en la elaboración y publicación de documentos como "Recomendaciones Bioéticas desde el enfoque de derechos humanos en Pandemia COVID19" (abril de 2020) en el cual se destaca que en medio de la pandemia lo urgente no tape o suprima lo esencial y lo importante que son los derechos y libertades de las personas. En ese documento ${ }^{3}$ se proponen principios bioéticos como la equidad, la responsabilidad, la flexibilidad temporalidad y la transparencia a la hora de tomar decisiones y programar las acciones a seguir. Se plantean ejes, como la planificación proactiva con 
una agenda de trabajo, asignación de recursos, prevención, optimización de recursos y beneficios, tratamientos, triaje clínico, atención de calidad, comunicación eficaz.

En el contexto de pandemia, que tocó vivir y que sigue en estos días, los derechos individuales se vieron limitados o acotados para beneficiar a la sociedad, pero esto nunca puede ser una excusa para atropellos u olvidos. Las distintas situaciones que se suscitaron mostraron la necesidad de priorizar el cuidado y acompañamiento humanizado y de calidad en todo momento y lo importante de velar por la calidad de vida de las personas, y procurar las despedidas con un duelo adecuado.

El aporte de los cuidados paliativos cobró gran importancia y nuevamente dejó en evidencia que estos cuidados es una materia pendiente en nuestro país. Estas situaciones motivaron que se elabore un documento en consonancia con colegas del resto del mundo sobre "Pautas para el acompañamiento de pacientes en situación de últimos días (SUD)"y "pacientes con condiciones especiales en contexto de pandemia por COVID19" (publicado en septiembre de 2020) para favorecer en lo posible el acompañamiento y las despedidas.

El desafío como Área de bioética fue poder tomar decisiones que sean racionales y prudentes en medio de situaciones de gran incertidumbre, buscando causar el menor daño posible a las personas: pacientes familias y equipos de salud. Trabajar para que las decisiones sean científicamente justificadas y moralmente aceptables.

La prioridad siempre es el cuidado de los vulnerados y los vulnerables, garantizar los derechos de las personas y procurar la mejor calidad de vida posible en todo momento. Todos estos valores nutren y justifican una bioética comprometida y de allí el rol preponderante que el Área de Bioética busca tener, aunque a veces es olvidada en las decisiones políticas (Toledo, 2020).

A lo largo de 20 años nos queda seguir aprendiendo de lo vivido, con la conciencia que hay mucho por hacer y un largo camino por recorrer.

\section{REFERENCIAS BIBLIOGRÁFICAS}

- Tealdi, Juan Carlos; Mainetti, José A. Los Comités Hospitalarios de Ética (1990)., Bol of Sanit Panam, 108, (5-6): 431-438.

- Toledo, A. F. (2020). El Derecho de la Salud en la gestión pública. La experiencia del Área de Bioética del Ministerio de Salud de la Provincia de Córdoba (Argentina) en algunas aplicaciones de este Derecho. Revista Derecho y Salud, Universidad Blas Pascal, 4(4), $17-$ 27. https://doi.org/10.37767/2591-3476(2020)01 